\title{
NATIONAL INSTITUTE OF JUSTICE
}

\section{FIVE THINGS ABOUT}

\section{SUBSTANCE USE INTERVENTIONS}
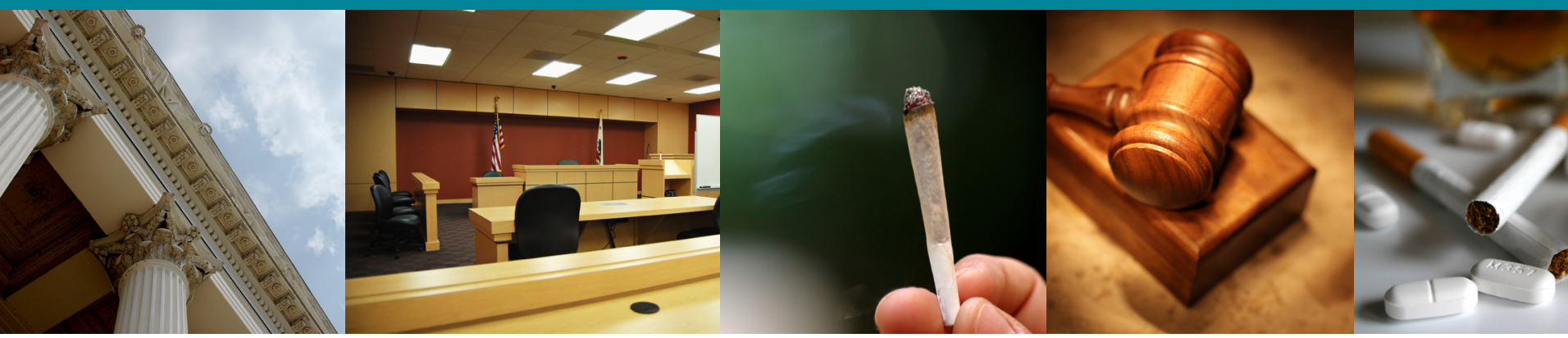

Substance use disorders, which include substance dependence and abuse, have a tremendous impact on individuals, families, and communities.

\section{The five statements below are based on practices rated by CrimeSolutions. ${ }^{1}$}

\section{Medication-assisted treatment is effective for reducing opioid dependence.}

Medication-assisted treatments for opioid dependence are designed to help patients with opioid addictions alleviate withdrawal symptoms, reduce or suppress opioid cravings, and reduce the illicit use of opioids.

Based on a review and rating by CrimeSolutions of multiple meta-analyses, medication-assisted treatments for individuals with opioid dependence have been found to be effective in reducing opioid dependence.

Medication-assisted treatments designed for persons with opioid dependence have been shown to be not effective for reducing the use of benzodiazepines or cocaine for persons who abuse those substances as well as opioids.

To learn more, review the practice profiles for:

- Opiate Maintenance Therapy for Dual Heroin-Cocaine Abusers

- Buprenorphine Maintenance Treatment

- Methadone Maintenance Therapy
2. Cannabis use disorder can be effectively treated using psychosocial interventions.

Psychosocial interventions may include many forms of therapy, such as cognitive behavioral therapy, contingency management, and relapse prevention to treat cannabis use disorder.

Based on a review and rating by CrimeSolutions of two meta-analyses composed of evaluations using randomized controlled trials, psychosocial interventions are effective for reducing the use of cannabis and the symptoms of dependence, and for increasing the prevalence of abstinence.

Review the practice profile Psychosocial Interventions for Cannabis Use Disorder to learn more.

3. Strategies that reward positive behavior and withhold rewards when undesired behavior is exhibited can reduce alcohol, tobacco, and illicit drug use.

Rewarding and withholding rewards as a means to affect behavior — called contingency management- is an intervention strategy designed to reduce substance use disorders. The overall goal is abstinence from substance use. 
Based on a review and rating by CrimeSolutions of two meta-analyses, both including multiple randomized controlled trials, participants in contingency management programs had lower rates of illicit drug use, alcohol use, and tobacco use than those who did not participate in such programs.

Review the practice profile Contingency Management Interventions for Substance Use Disorders to learn more.

\section{Short-term interventions can reduce alcohol use or related problems for adolescents and young adults.}

This practice seeks to reduce alcohol use or alcohol-related problems for adolescents and young adults via a short-term intervention (one to five sessions).

Based on a review and rating by CrimeSolutions of a meta-analysis, such interventions are effective for reducing alcohol consumption and alcohol-related problem outcomes for adolescents and young adults.

See the practice profile Brief Alcohol Interventions for Adolescents and Young Adults to learn more.

\footnotetext{
${ }^{1}$ As defined by CrimeSolutions, a practice is a general category of programs, strategies, or procedures that share similar characteristics with regard to the issues they address and how they address them. Practice profiles tell us about the average results from multiple evaluations of similar programs, strategies, or procedures.
}

\section{Juvenile drug courts do not have an effect on drug and alcohol offense recidivism or future drug use.}

Juvenile drug courts are dockets within juvenile courts for cases involving youth who have abused substances and are in need of specialized treatment services. The focus is on providing treatment to eligible youth who are involved with drugs, with the goal of reducing recidivism and substance abuse.

Based on a review and rating by CrimeSolutions of two meta-analyses, juvenile drug courts have no significant effect on the incidence of drug-related offenses or substance use.

See the practice profile Juvenile Drug Courts to learn more.

\section{CrimeSolutions}

CrimeSolutions helps practitioners and policymakers understand what works in justice-related programs and practices.

Crimesolutions is funded by the National Institute of Justice and the Office of Juvenile Justice and Delinquency Prevention (OJJDP). Programs and practices profiles related to juveniles also appear on OJJDP's Model Programs Guide. 